Intersections

Canadian Journal of Music

Revue canadienne de musique
Intersections

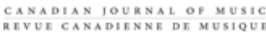

\title{
"A garland on Pierre Boulez ». Quelques considérations sur Pour le Dr. Kalmus (1969/2005)
}

\section{Brice Tissier}

Volume 34, numéro 1-2, 2014

URI : https://id.erudit.org/iderudit/1030874ar

DOI : https://doi.org/10.7202/1030874ar

Aller au sommaire du numéro

Éditeur(s)

Canadian University Music Society / Société de musique des universités canadiennes

ISSN

1911-0146 (imprimé)

1918-512X (numérique)

Découvrir la revue

Citer cet article

Tissier, B. (2014). «A garland on Pierre Boulez ». Quelques considérations sur Pour le Dr. Kalmus (1969/2005). Intersections, 34(1-2), 153-179.

https://doi.org/10.7202/1030874ar
Résumé de l'article

Retirée après sa création en 1969, la pièce Pour le Dr Kalmus de Pierre Boulez a fait en 2005 une surprenante réapparition. On ignore encore les raisons qui ont décidé Boulez à réviser cette partition. On ignore d'ailleurs presque tout la concernant. Il est toutefois possible aujourd'hui, à la lumière des quelques sources manuscrites conservées et de la nouvelle partition éditée, de faire plusieurs constatations historiques, philologiques et analytiques sur cette oeuvre certes secondaire dans le corpus boulézien, mais non dénuée d'intérêt, et composée à un moment charnière de sa production musicale et de son évolution esthétique.
Copyright @ Canadian University Music Society / Société de musique des universités canadiennes, 2015
Ce document est protégé par la loi sur le droit d'auteur. L'utilisation des services d’Érudit (y compris la reproduction) est assujettie à sa politique d'utilisation que vous pouvez consulter en ligne.

https://apropos.erudit.org/fr/usagers/politique-dutilisation/ 


\section{"A GARLAND ON PIERRE BOULEZ". QUELQUES CONSIDÉRATIONS SUR POUR LE DR. KALMUS (1969/2005)}

\section{Brice Tissier}

En 2005, année du quatre-vingtième anniversaire de Pierre Boulez, une petite pièce a fait une surprenante réapparition. Pour le Dr. Kalmus n'avait plus été entendue depuis 1969 et semblait destinée à n'être plus qu'une simple référence dans le catalogue des œuvres du compositeur du Marteau sans maître, à l'image du Crépuscule de Yang Kouei-fei et de Symphonie mécanique. Pourtant, Pour le Dr. Kalmus fut bel et bien révisée en 2005 et éditée chez Universal Edition. Plusieurs ensembles ont depuis mis l'œuvre à leur répertoire'

\section{Tableau 1}

\begin{tabular}{|c|c|c|}
\hline Ensemble & date & lieu \\
\hline $\begin{array}{l}\text { Oslo Filharmoniske Ork- } \\
\text { esterOslo Filharmoniske } \\
\text { OrkesterOslo Filharmoniske } \\
\text { Orkester }\end{array}$ & 4 octobre 2006 & Parkteatret, Oslo \\
\hline Ensemble Recherche & 10 mars 2007 & Théâtre Marni, Bruxelles \\
\hline Argento Chamber Ensemble & $\begin{array}{l}19 \text { novembre } 2008 \text {, } \\
5 \text { mai } 2009 \\
12-26 \text { septembre et } 9 \text { décembre } \\
2010\end{array}$ & $\begin{array}{l}\text { Djakarta } \\
\text { Merkin Concert Hall, NYC } \\
\text { Austrian Cultural Forum, NYC }\end{array}$ \\
\hline Ensemble Garage & 13 et 19 mai 2011 & Musiktriennale Köln 2011 \\
\hline Collegium novum Zurich & $\begin{array}{l}26 \text { novembre et } 1^{\text {er }} \text { décembre } \\
2011\end{array}$ & Haus Konstruktiv, Zurich \\
\hline Ensemble InterContemporain & 24 janvier 2012 & Villa Médicis, Rome \\
\hline
\end{tabular}

Dans quel but Boulez a-t-il révisé cette œuvre? Pourquoi avait-elle été retirée pendant plus de trente ans? On l'ignore encore. Et l'on ignore de surcroît tout ce qui la concerne : Boulez ne l'a jamais évoquée, elle n'est mentionnée ni dans ses écrits ni dans ses interviews, et il ne l'a jamais dirigée. Il n’a jamais souhaité non plus la présenter par un concert lecture ou un communiqué. Il était donc nécessaire de dévoiler cette œuvre méconnue à la lumière des sources qui sont aujourd'hui disponibles et d'en cerner les caractéristiques et les enjeux à travers plusieurs considérations qui tenteront de pénétrer cette partition encore mystérieuse.

1 Tableau 1. L'ensemble InterContemporain a également réalisé en 2012 un enregistrement de la pièce, à paraître dans la future édition intégrale des œuvres de Boulez chez Deutsche Grammophon. 


\section{L'ART DE L'HOMMAGE BOULÉZIEN}

L'hommage est un art obligé pour tout compositeur - anniversaire, jubilé, décès. Les bibliothèques regorgent de ces pages volantes écrites à la va-vite, généralement un court fragment manuscrit, avec dédicace, extrait - ou anticipé - d'une œuvre majeure. Boulez ne dédaignera jamais cet exercice, et certaines pages donneront de grandes œuvres. Messages (1974) ${ }^{2}$, pour les 80 ans de Paul Sacher, deviendra Messagesquisse. Anthèmes (1990)3 3 , pour les 80 ans d'Alfred Schlee, deviendra Anthèmes 2. Les différentes versions d'...explosantefixe... (1972) ${ }^{4}$, pour la mort de Stravinski, engendreront une pléiade d'ouvres éponymes et marginales 5 .

Dans d'autres cas, ces brefs hommages furent intégrés dans des œuvres de plus grande envergure. Les 7 interjections à Pierre Souvtchinski ${ }^{6}$ ont donné les sections impaires de Rituel, et Domaine-Epigraphe-Texte-Dédicace pour le 80 anniversaire de Stravinski (Baltensperger 1990, p. 15-22) constitue désormais l'ensemble des inserts de Don (à partir de 1962).

D’autres, enfin, sont restées lettres plus ou moins mortes, ou en devenir, telles la Petite dérive (1998) pour les 90 ans d'Elliott Carter ${ }^{7}$ ou Dérive 3 (1997) pour les 70 ans de Michael Gielen ${ }^{8}$.

Pour le Dr. Kalmus, quant à elle, fut écrite et dédiée à l'éditeur londonien des œuvres de Boulez. Alfred A. Kalmus (1889-1972) était déjà directeur de Universal Edition ${ }^{9}$ lorsqu'il fut forcé d'émigrer à Londres en 1936, fuyant Vienne et sa politique répressive. Il y crée la branche londonienne de l'éditeur et diffuse les créateurs prometteurs : Berio, Stockhausen, Birtwistle, Boulez ${ }^{10} \ldots$ Le 22 avril 1969, onze d'entre eux lui rendent hommage lors d'un concert: A Garland for Dr. K., au Queen Elisabeth Hall ${ }^{11}$. Outre Boulez, y participent David Bedford, Hugh Wood, Richard Rodney Bennett, Luciano Berio, Cristobal Halffter, Roman Haubenstock-Ramati, Harrison Birtwistle, Bernard Rands et Henri Pousseur. Le style est libre mais deux imposés accompagnent la commande :

2 Messages, édité en facsimilé dans le recueil des 12 hommages à Paul Sacher, $\odot$ Copyright 1980 by Universal Edition A.G., Wien/UE 17182.

3 Version abrégée de la pièce éponyme élaborée à la demande de Yehudi Menuhin pour le concours de la ville de Paris; un enregistrement de cette première étape existe dans From Vienna, Arditti String Quartet, Montaigne MO 782027, 1994.

4 «In Memoriam: Igor Stravinsky. Canons \& Epitaphs, Set $2 »$. Tempo, new series, no. 98 (1972), p. $13-26$.

5 Voir la thèse de Paolo Dal Molin 2007.

6 Bibliothèque Nationale de France, section musique, Ms. 21611.

7 Le manuscrit facsimilé est consultable sur Internet : http://archive.org/details/PetiteDeriveEnchoPierreBoulez (consulté en février 2013).

8 Fondation Paul Sacher (Mappe K, Dossier 5a). En réalité la première page redéployée d'Initiale (1987) composée pour la Menil Foundation, inédite, créée à Houston le 6 avril 1987 (Mappe L, Dossier 1a); un enregistrement est conservé à la médiathèque de l'Ircam.

9 Tradition familiale : son jeune frère Edwin F. Kalmus (1893-1989) avait fondé en 1926 à New York la maison d'édition musicale qui porte depuis son nom.

10 Boulez cumule les paradoxes nationaux : compositeur français, il déclare ses œuvres à la GEMA - l'équivalent de la SACEM - en Allemagne, et est édité par la branche anglaise d'une maison d'édition autrichienne.

11 Le concert comprenait également les créations mondiales de Eight Songs for a Mad King de Peter Maxwell Davies, and Linoi II de Harrison Birtwistle. 
une durée brève - entre 1' et 3'30 - et une instrumentation circonscrite à une formation instrumentale précise (en gras dans le tableau 2) : un quintette flûte, clarinette, violon (ou alto), violoncelle et piano. Sept ans après l'évènement, un enregistrement des onze hommages au Dr. Kalmus sera réalisé en édition limitée ${ }^{12}$ : A Garland for Dr. K., compositions for the 8oth birthday of Dr. Alfred $A$. Kalmus. Cet enregistrement nous permet de dresser une liste précise et référencée de ces onze hommages (Tableau 2):

Tableau 2

\begin{tabular}{|c|c|c|c|}
\hline Compositeura & titre & Effectif instrumental & durée \\
\hline D. BEDFORD & A Garland for Dr. K. & $\begin{array}{l}\text { fl, cl (basse), vl (vla), vc, pno, } \\
\text { clav. }\end{array}$ & $3{ }^{\prime} 29$ \\
\hline H. WOOD & A Garland for Dr. K. & fl, cl, vl, vc, pno. & $1 ' 09$ \\
\hline P. BOULEZ & Pour le Dr. Kalmus & fl, cl (ut), vla, vc, pno. & 2'32 \\
\hline R. R. BENNETT & Impromptu & fl. & $2^{\prime} 48$ \\
\hline L. BERIO & $\begin{array}{l}\text { The modification and instrumen- } \\
\text { tation of a famous Hornpipe as } \\
\text { a merry and altogether sincere } \\
\text { homage to Uncle Alfred (Nach } \\
\text { Purcell) }\end{array}$ & fl, vla, vc, perc, clav. & $1 ' 04$ \\
\hline C. HALFFTER & Oda para felicitar a un amigo & fl en sol, cl (sib), vla, vc, perc. & $6{ }^{\prime} 37$ \\
\hline $\begin{array}{l}\text { R. HAUBENSTOCK- } \\
\text { RAMATI }\end{array}$ & Rounds & fl, cl, vla, vc, vibr, pno. & 2'37 \\
\hline H. BIRTWISTLE & Some petals from the garland & $\begin{array}{l}\text { fl picc, cl (sib), vla, vc, glock, } \\
\text { pno. }\end{array}$ & $2 ' 17$ \\
\hline K. STOCKHAUSEN & Für Dr. K. & fl, cl basse, vla, vc, perc, pno. & $4 ' 14$ \\
\hline B. RANDS & Monotone & fl, cl, vla, vc, perc, pno (clav). & $1 ' 35$ \\
\hline H.POUSSEUR & Echos II de votre Faust & fl, vc, pno. & 3'03 \\
\hline
\end{tabular}

a Dans l'ordre de l'enregistrement précédemment cité. Le 12e hommage, celui de Ernst Krenek, n'est pas musical en soi : il consiste en une partition en cercle, à réaliser soi-même, basée sur les permutations circulaires d'une mélodie créée d'après le mot-valise kalmusikal. « L'œuvre » ne fut pas réalisée et enregistrée, mais reproduite en facsimilé dans la pochette du disque-hommage avec la photographie d'une autre œuvre de Mary Bauermeister, alors compagne de Stockhausen et illustratrice de plusieurs couvertures de partition pour Universal Edition.

Certains de ces hommages furent immédiatement considérés comme œuvres à part entière, éditées et enregistrées : Für Dr. K. de Stockhausen, Echos II de votre Faust de Pousseur, ou Impromptu de Bennett. La participation de Boulez pose en revanche problème car la pièce enregistrée en 1976 est différente de celle jouée au Queen Elisabeth Hall. Le compte rendu du concert paru le 26 avril dans le Times rapporte en effet que «[...] Boulez supplies a clarinet solo with full of lively squeals» (Mann 1969a, p. 16). Boulez présente une seconde version de son hommage le 16 mai 1969 - date précise de l'anniversaire de Kalmus - lors d'un concert privé au domicile de l'éditeur. C'est cette seconde version qui sera gravée et considérée comme officielle pour A Garland for Dr. K. Le seul hommage écrit de Boulez sur Alfred A. Kalmus est un bref texte signé, conservé dans les archives de l'éditeur :

12 A Garland for Dr. K., compositions for the 8oth birthday of Dr. Alfred A. Kalmus, Londres, Universal Edition UE 15043-104, 1970. Hors commerce. 
Le Docteur Kalmus était pour moi à la fois une légende, un mythe et une personne si présente, si bienveillante qu'il m'était difficile de joindre ces deux aspects en une même individualité. Il avait connu personnellement les musiciens que j'admirais et qui m’avaient formé. Cependant, il s'intéressait de près à une nouvelle génération passablement turbulente. Face à la turbulence affichée, il gardait un calme d'observateur cultivé et perspicace. Ces qualités rares faisaient que l'on le respectait, bien sûr, mais que le respect, loin d'empêcher l'affection, l'aidait et la fortifiait. Aussi paradoxal que cela puisse se formuler, son calme transmettait avec opiniâtreté la tradition de l'aventure ${ }^{13}$.

L'œuvre ayant été retirée, l'enregistrement de 1976 fut, pendant plus de trente ans, la seule trace matérielle de l'hommage de Boulez. On pourrait croire que l'imposé soit la cause de ce retrait, or l'effectif instrumental n'était, au contraire, pas pour déplaire à Boulez.

\section{L'instrumentation de Pour le Dr. Kalmus : une constante BOULÉZIENNE}

L'instrumentarium était imposé car, en 1969, l'ensemble chargé de l'exécution des douze hommages à Kalmus était The Fires of London, un ensemble dédié spécifiquement à l'interprétation de la musique contemporaine, fondé à Londres en 1965 par Harrison Birtwistle, Alan Ray Hacker et Stephen Pruslin. Lorigine de cet ensemble, et donc sa nature et sa composition, sont liées au Pierrot Lunaire de Schoenberg et à son effectif spécifique : soprano, flûte, clarinette, violon, violoncelle, piano; d'où le nom originel de l'ensemble : Pierrot Players. Depuis 1967, Peter Maxwell Davies et Harrison Birtwistle se partageaient la direction de l'ensemble, ce qui explique notamment l'importance des Fires of London dans l'exécution des œuvres propres de Davies. Ce lien entre l'ensemble et le compositeur se renforce cependant à partir de 1970, date à laquelle Birtwistle quitte le groupe, laissant Davies seul aux commandes. The Fires of London sera finalement dissous en 1987, lors d'un concert anniversaire fêtant les vingt ans de l'ensemble. Les compositions de Boulez d'effectif comparable montrent une certaine proximité avec les Pierrot Players (Tableau 3).

13 Hommage conservé aux archives de Universal Edition et dans les archives de l'Ensemble InterContemporain, s.l.n.d. 
Tableau 3

\begin{tabular}{|c|c|c|c|c|c|}
\hline Éclat (1965) & $\begin{array}{c}\text { Pour le } \\
\text { Dr. Kalmus } \\
\text { (1969) }\end{array}$ & $\begin{array}{c}7 \text { Interjections }^{\mathrm{a}} \\
(1972)\end{array}$ & $\begin{array}{c}\text {...explosante- } \\
\text { fixe...b } \\
(1972-1973)\end{array}$ & Dérive (1984) & $\begin{array}{c}\text { Petite dérivec } \\
\text { (1990) }\end{array}$ \\
\hline \multirow[t]{2}{*}{ Flûte } & Flûte & Flûte & Flûte & Flûte & Flûte \\
\hline & & Hautbois & & & \\
\hline Cor anglais & Clarinette ut & $\begin{array}{l}\text { Clarinette } \\
\text { basse }\end{array}$ & Clarinette & Clarinette la & Clarinette sib \\
\hline \multicolumn{2}{|l|}{ Trompette en ut } & & Trompette & & \\
\hline \multicolumn{6}{|l|}{ Trombone } \\
\hline & & & Violon & Violon & Violon \\
\hline Alto & Alto & Alto & Alto & & Alto \\
\hline Violoncelle & Violoncelle & & Violoncelle & Violoncelle & \\
\hline \multicolumn{6}{|l|}{ Glockenspiel } \\
\hline \multirow[t]{2}{*}{ Vibraphone } & & & Vibraphone & Vibraphone & Vibraphone \\
\hline & & & & & Marimba \\
\hline \multicolumn{6}{|l|}{ Mandoline } \\
\hline \multicolumn{6}{|l|}{ Guitare } \\
\hline Cymbalum & & Cymbalum & & & \\
\hline \multicolumn{6}{|l|}{ Cloches tubes } \\
\hline Piano & Piano & Piano & & Piano & \\
\hline \multicolumn{6}{|l|}{ Célesta } \\
\hline harpe & & Harpe & Harpe & & \\
\hline
\end{tabular}

Flûte et clarinette sont omniprésentes. Le hautbois en revanche n'est pas un instrument «boulézien». On remarque une translation amusante de l'usage de l'alto vers le violon (annonçant l'Anthèmes de 1991), ainsi que l'abandon progressif du piano au profit du vibraphone - ce dont témoigne par ailleurs l'évolution des esquisses d'...explosante-fixe... entre 1970 et $1974^{14}$.

Ce n'est donc pas par rejet de l'effectif imposé que l'hommage proposé par Boulez en avril 1969 n'utilisait que la clarinette. Probablement le manque de temps n'aura-t-il pas permis au compositeur de mener à bien son projet de partition, reculant ainsi la création de l'hommage de quelques semaines. L'étude philologique de Pour le Dr. Kalmus vient par ailleurs renforcer cette hypothèse.

\section{UN PROBLÈME PHILOLOGIQUE}

La méconnaissance actuelle de Pour le Dr. Kalmus est due à l'insuffisance des sources musicales disponibles. Le statut de petite œuvre de circonstance peut

14 Certains instruments, utilisés en musique de chambre, connaîtront une postérité boulézienne intéressante : outre le vibraphone fondamental pour les Improvisations sur Mallarmé, le marimba sera au cœur des préoccupations pour le Duo (marimba et contrebasse) issu de Domaines en 1982; cymbalum, vibraphone, piano et harpe seront les principaux solistes de Répons. 
en partie expliquer l'absence d'esquisses, de schémas ou de notes préparatoires, mais ne couvre pas l'absence totale de documents.

À ce jour, nous disposons de deux sources :

Un manuscrit autographe dédicacé : six pages de musique format italien 37 x $26 \mathrm{~cm}, 14$ portées (provisoirement publié, mais retiré : Pour le Dr. Kalmus, Kopie hergestellt von Originalverlag, (c) Copyright 1969 by Universal Edition (London) Ltd., London/UE 30898, 6 p.). Il s'agirait logiquement du manuscrit offert à A. Kalmus à l'occasion du concert privé de mai 1969, et resté probablement la possession de la famille Kalmus. On peut s'interroger sur la nature de ce manuscrit : il s'agit d'une mise au net, copiée sur un carnet relié à spirales - et non sur des pages volantes, comme Boulez en a souvent l'habitude. Ce manuscrit ne ressemble pas aux manuscrits premiers de Boulez, beaucoup plus «bâclés» et raturés. Les quelques corrections reportées sur le document sont vraisemblablement le fruit d'une réflexion secondaire. Il pourrait ainsi s'agir ici d'un manuscrit rédigé d'après des sources ou des matériaux préexistants, c'est-à-dire d'après une base ou une œuvre préalable. Cette déduction expliquerait l'absence d'esquisses, à l'image de l'élaboration de la première Improvisation sur Mallarmé, constituée à la va-vite en 1957 en collectant des matériaux issus de la musique radiophonique du Crépuscule de Yang Kouei-fei (une ligne vocale constituée - hauteurs et rythmes - entrecoupée de deux des Notations pour piano recyclées).

La partition éditée en 2005 chez Universal Edition (Improvisé - pour le Dr. $K$., pour piano et quatre instruments, (c) Copyright 2005 by Universal Edition A.G., Wien/UE 33544, 2005, 12 p.) est désormais disponible à la location. Cette dernière comporte plusieurs différences notables, qui ne sont pas réductibles aux quelques corrections et ajouts de tempi et nuances officiellement invoqués pour justifier cette nouvelle édition. Il semblerait logique de croire que cette correction serait le fruit du travail et des corrections préalables à une programmation de l'œuvre, toutefois aucune interprétation n'est recensée avant $2006^{15}$. La raison la plus logique serait donc le parallèle entre les circonstances de cet hommage $-8 \mathrm{o}^{\mathrm{e}}$ anniversaire de Alfred A. Kalmus - et celles de son édition $8 \mathrm{o}^{\mathrm{e}}$ anniversaire du compositeur.

Aucun document n'a encore été déposé à la Fondation Paul Sacher, récipiendaire de tous les manuscrits de Boulez. Comme nous l'avons écrit, le statut d'hommage peut vraisemblablement expliquer l'absence d'esquisses et de schémas préparatoires. On ne peut exclure non plus la possibilité que rien n'ait été conservé par le compositeur ${ }^{16}$.

15 La création américaine par l'Argento Chamber ensemble en 2009 est consultable sur le site Internet Youtube : http://www.youtube.com/watch?v=wvQOfWolOgA. (Consulté en janvier 2013.); de même qu'un extrait du travail de répétition en présence de Boulez : http://www.youtube.com/ watch?v=p36pgSK6qOg. (Consulté en janvier 2013.)

16 Il serait également possible que Boulez ait souhaité conserver quelques documents : à ce jour il conserve toujours l'ensemble des manuscrits et esquisses de Répons, ...explosante-fixe... (version 1991-93) et sur Incises. 
Aucun document manuscrit n'est non plus conservé aux archives de Universal Edition à Vienne ${ }^{17}$. Outre la partition éditée en 2005, seuls deux documents y sont référencés :

- un court texte de Boulez précédemment cité, dactylographié sur une page volante et signé de la main du compositeur

- un texte de présentation de Pour le Dr. Kalmus rédigé par Therese Muxeneder (archiviste au Centre Arnold Shönberg de Vienne).

De ce fait, l'étude de Pour le Dr. Kalmus doit encore se limiter à la comparaison entre les deux partitions connues de l'œuvre.

\section{LES DEUX PARTITIONS COMPARÉES : DE 1969 À 2005}

La partition publiée en 2005 ne constitue pas concrètement une nouvelle version : outre l'ajout de tempi et de quelques indications de jeu, les modifications par rapport au manuscrit dédicacé de 1969 sont minimes. La seule modification majeure est la substitution de la clarinette en $u t$, remplacée par une clarinette en sib.

D'autres modifications ont pour but d'«officialiser» l'œuvre. Par exemple, le manuscrit de 1969 ne comportait aucun titre. Contrairement à ses confrères,
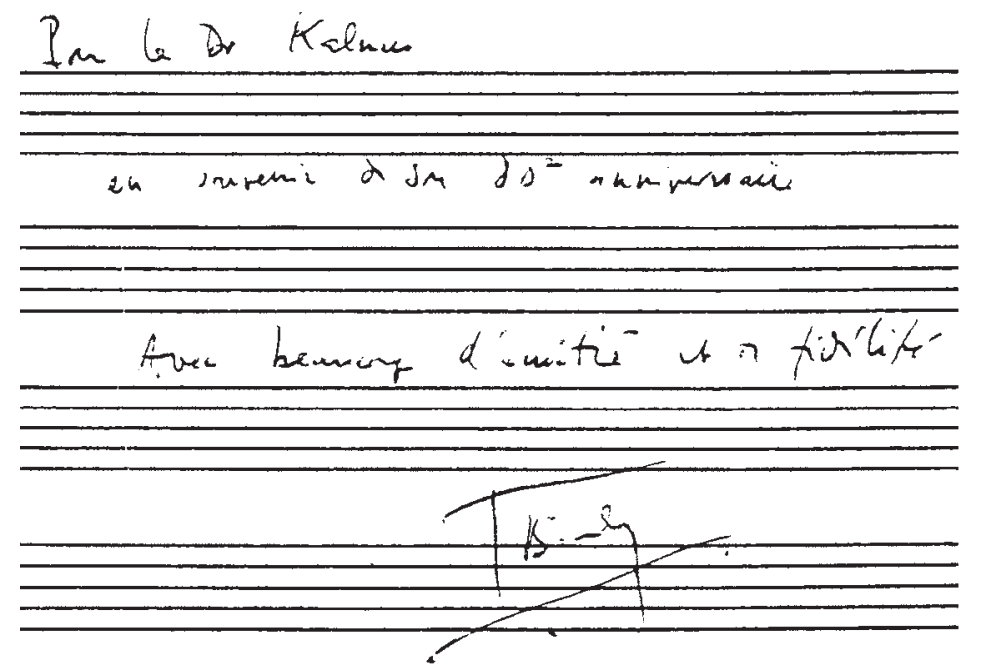

Exemple 1: dédicace de Boulez au Dr. Kalmus. « Pour le Dr. Kalmus, en souvenir de son 8 oe anniversaire. Avec beaucoup d'amitié et de fidélité. P. Boulez », dédicace de la dernière page du manuscrit original de 1969, op. cit., p. 6.

Boulez - tout comme Stockhausen - rejette tout titre personnel, ainsi que celui plus général de l'évènement ( $A$ garland for Dr. K. repris par Bedford et

17 Les archives de la branche londonienne de Universal Edition sont désormais transférées automatiquement aux archives historiques de la maison Universal, à Vienne. 
Wood). La dénomination de sa partition est extraite de la dédicace finale - ce qui explique par ailleurs l'emploi du nom complet du destinataire, et non sa simple initiale ${ }^{18}$.

La révision de 2005 change deux aspects de ce 'faux titre' : Boulez aligne sa pièce sur les autres hommages à Kalmus en gommant le nom du docteur, et l'intitulé est complété : Improvisé - pour le Dr. K. Le terme «improvisé» est surprenant de la part de l'auteur des trois Improvisations sur Mallarmé:

L'improvisation, telle que je l'entends, est irruption (Einbruch) dans la musique d'une dimension libre. Dans l'exécution d'une œuvre orchestrale traditionnelle, les musiciens sont dépendants, aussi bien du chef d'orchestre que des lois d'un jeu collectif réglé avec précision, et qui ne peut être transgressé. Dans l'improvisation, par contre, deux données s'assouplissent : la forme elle-même, et où les relations entre instruments doivent jouer. [...] Jusqu'à une époque récente, la forme était définie de manière précise dans tous ses détails. On avait un langage musical établi à partir des hiérarchies acceptées, dont dépendaient figures et agencements. Aujourd'hui, le langage se bâtit pour l'essentiel sur des phénomènes relatifs, c'est pourquoi la forme doit elle aussi être relative. Autrement dit : il faut y introduire des éléments qui la modifient d'une exécution à l'autre, et empêchent qu'une œuvre soit jouée deux fois exactement de la même manière ${ }^{19}$.

Or ni la version de 1969 (contemporaine de Multiples et de Domaines III), ni la révision de 2005 ne possèdent le moindre degré d'ouverture, tant sur la forme que sur l'interdépendance entre les cinq instruments. Nous y reviendrons.

Plusieurs modifications déjà reportées sur le manuscrit en 1969 sont désormais officialisées. Par exemple, deux mesures de la section centrale (appelonsles $\mathrm{m}$. $34 \mathrm{~b}$ et c) sont barrées, la rustine entre les mesures 34 et 35 étant assurée par un si bécarre lié au piano et à la flûte m. $34^{20}$ (Exemple 2).

On peut constater sur cet extrait plusieurs autres corrections mineures, principalement dans l'agencement des hauteurs. (Tableau 3)

La partition de 2005 révèle que d'autres corrections ont été effectuées ultérieurement, et donc ne sont pas matérialisées sur la partition de 1969. (Tableau 4)

Cette seconde campagne de corrections n'influe pas sur l'écriture harmonique, excepté une modification conséquente dans le retour de la cadence initiale de piano avant la coda, où Boulez convertit la ligne mélodique en blocs

18 Situation qui n'est pas sans rappeler celle du choix du titre de Cummings ist der Dichter.

19 Boulez P., «Construire une improvisation», conférence à Strasbourg (1961) sur la deuxième Improvisation sur Mallarmé (trad. Jean-Louis Leleu, révisée par l'auteur en 1981), reprise dans Points de repère I/Imaginer 1995, p. 445-446.

20 Ce type de censure est courant dans les manuscrits bouléziens, par exemple dans le conducteur laissé à l'état de manuscrit de Transitoire VII. La présence de telles ratures au sein du discours est importante : elle indique une écriture non fondée sur des permutations ou des jeux sériels dans le style des années 50, laquelle n'admettrait pas la suppression d'une partie des trajectoires sérielles. 


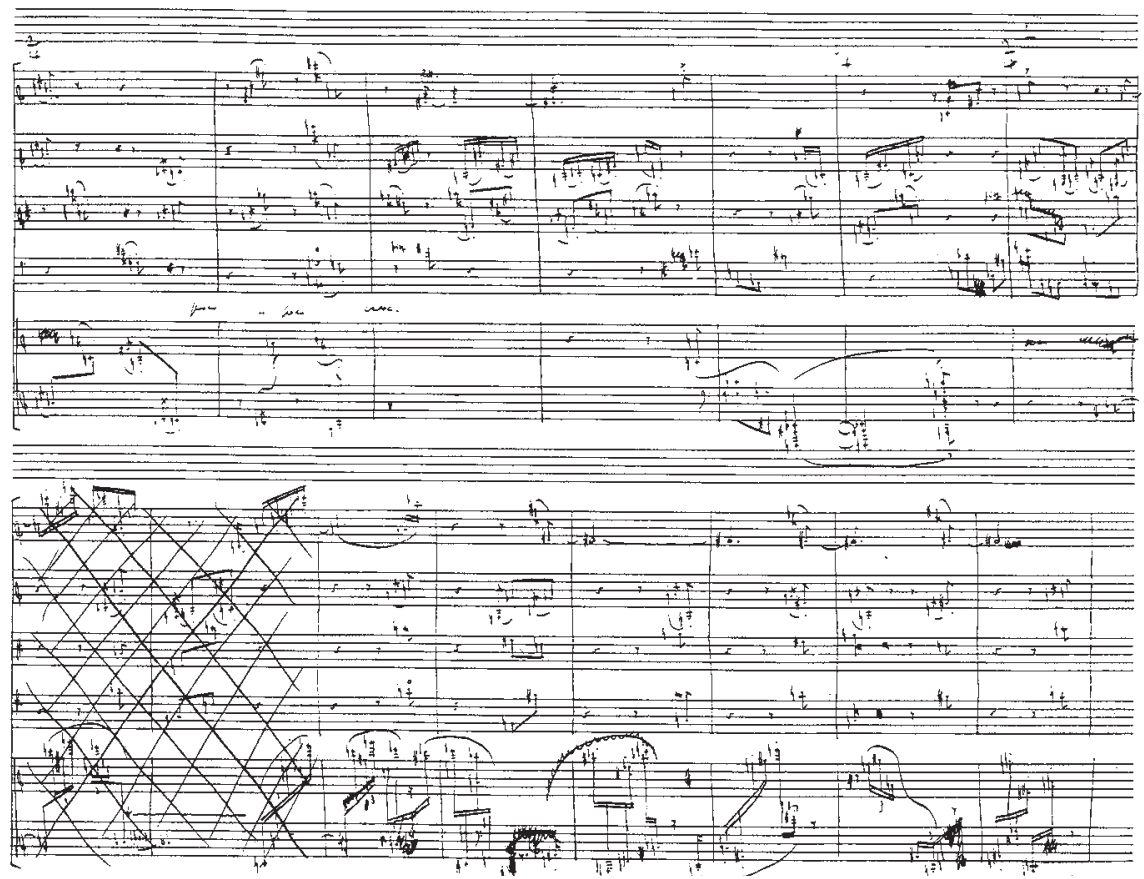

Exemple 2 : corrections mineures et mesures coupées sur le manuscrit de 1969. Pour le Dr. Kalmus, op. cit., p. 3.

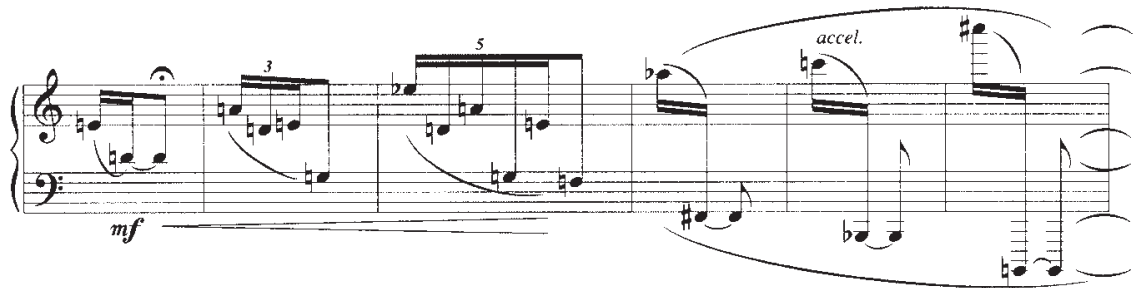

Libre $d=116$

accel.

molto

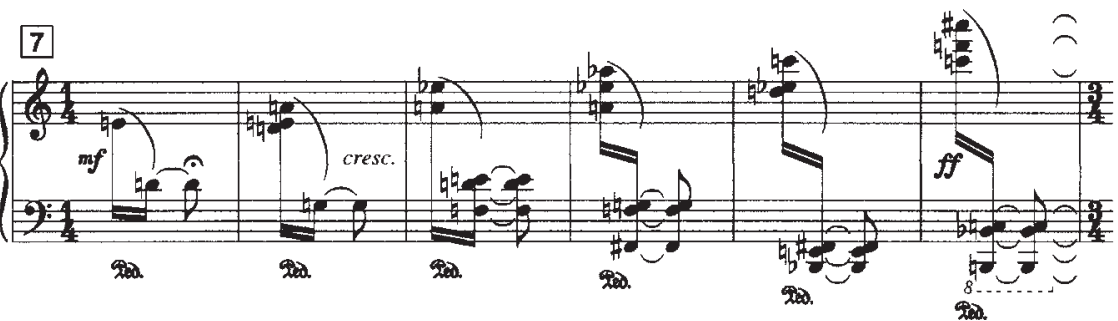

Exemples 3 a et b: retour de la cadence de piano (versions 1969 et 2005). Pour le Dr. Kalmus, op. cit., p. 5-6 ; Improvisé - pour le Dr. K., op. cit., p. 12. 
harmoniques ${ }^{21}$ avant d'homogénéiser la séquence en rajoutant des blocs harmoniques aux trois autres mesures (Exemples $3 \mathrm{a}$ et $\mathrm{b}$ ).

Cette ligne mélodique n'est pas la seule à connaître une récurrence, car Boulez joue sur l'ensemble des éléments constitutifs de Pour le Dr. Kalmus, influençant ainsi la forme générale de l'œuvre. Il devient alors nécessaire d'identifier les matériaux et motifs constitutifs. Parmi ces matériaux, deux ont vocation d'hommage et doivent être pré-étudiés.

\section{DEUX CITATIONS INATTENDUES}

Les corrections pour l'édition de 2005 ont masqué deux clins d'œil anecdotiques, à deux endroits stratégiques. Au chiffre 6 , une cassure nette est visible avant la grande section centrale. Venue de nulle part, la flûte énonce en trilles le début de la partie vocale de la première Improvisation sur Mallarmé. L'attribution de cette mélodie à la flûte n'est du reste pas anodine : génétiquement, la ligne vocale de l'Improvisation était issue de Strophes (1957) pour flûte seule, rétrogradée par rapport à la partition initiale ${ }^{22}$. Sur le manuscrit de 1969, Boulez avait signé son hommage en reportant, au-dessus de la ligne de flûte puis de la clarinette, le texte du Vierge, le vivace et le bel aujourd'hui. L'édition de 2005 a supprimé cette référence (Exemple 4).

De même, au chiffre 1, deux enchaînements d'accords crescendo paraissent anodins dans la révision de 2005 (Exemples $5 \mathrm{a}$ et b).

Sur le manuscrit de 1969, Boulez appose, ici encore, deux mots - un pour chaque enchaînement - au-dessus de la partie de clarinette en $u t$, désormais en $s i b$ : «Happy birthday !» Cette mélodie de la clarinette ne laisse aucune ambiguïté : ré-mi-ré-sol-fa\#, c'est-à-dire le thème de "Happy birthday to you » immortalisé par Marilyn à JFK sept ans auparavant (Exemple 6).

Cette allusion fait écho aux contributions de Wood et Birtwistle, qui intégraient également cette citation. Les positions de ces deux hommages - «Le Vierge, le vivace et le bel aujourd'hui» et "Happy birthday to you» - ne sont pas aléatoires dans la forme de l'œuvre : elles permettent une mise en évidence de la structure en arche de Pour le Dr. Kalmus (Exemple 7), forme dont la simplicité est probablement due à une élaboration rapide mais dont on peut également trouver l'origine dans un autre projet boulézien.

\section{UNE FORME DÉJÀ RENCONTRÉE}

La forme de Pour le Dr. Kalmus tient beaucoup d'Éclat. Dans un article en 1993 (Piencikowski 1993, p. 51-68), Robert Piencikowski avait mis en évidence les structures, ainsi que les enjeux matériels d'Éclat, à savoir la refonte d'une

${ }^{21}$ L'engendrement des blocs harmoniques de 2005 est obtenu par la verticalisation des arabesques de 1969, elles-mêmes obtenues par le report des sons du geste précédent sur le suivant. Le procédé, circonscrit par Boulez aux trois premiers blocs en 1969, est étendu à l'ensemble des gestes (des notes originales aux extrêmes). Au passage, signalons les éléments rajoutés : tempo, caractère et indications de pédale.

22 Strophes était elle-même une refonte de certains passages de la musique de scène pour l'Orestie (1955). Voir Piencikowski 1993, p. 51-68. 


\section{Tableau 3}

\begin{tabular}{|c|c|}
\hline mesure & Corrections effectuées sur le manuscrit de 1969 \\
\hline m. 2 & - pno : séparation des doubles croches et de la croche. \\
\hline m. 8 & - cl : ronde descendue d'une seconde majeure (rédaction en $u t)$. \\
\hline m. 9 & $\begin{array}{l}\text { - alt : indication pizz supprimée. } \\
\text { - pno : ré-mib descendus d'une octave. }\end{array}$ \\
\hline m. 11 & - vc : 5 croches séparées en $3+2$ \\
\hline m. 17 & - $\mathrm{fl}: 4$ croches séparées en $2+2$ \\
\hline m. 24 & - pno : liaison supprimée sur le dernier quintolet. \\
\hline m. 25 & - pno : indication mf supprimée. \\
\hline m. 26 & - barre de mesure supprimée au $4^{\mathrm{e}}$ temps (mesure passée en 5/4). \\
\hline m. 28 & - pno : appoggiature passée à la main gauche. \\
\hline m. 30 & - $\mathrm{cl}: 2^{\mathrm{e}}$ double croche supprimée. \\
\hline m. 31 & - vc : appoggiature supprimée. \\
\hline m. 33 & $\begin{array}{l}\text { - fl : do octavié. } \\
\text { - vc : } 3^{e} \text { croche }(d o) \text { supprimée. }\end{array}$ \\
\hline m. 34 & $\begin{array}{l}\text { - } 1_{\text {ère }} \text { croche : la devient } m i ; 3^{\mathrm{e}} \text { croche : do devient la. } \\
\text { - alt : deux premières croches changées. } \\
\text { - pno : si lié passé à la main gauche. }\end{array}$ \\
\hline m. $34 \mathrm{~b} / \mathrm{c}$ & - supprimées. \\
\hline m. 35 & $\begin{array}{l}\text { - pno : } 4 \text { doubles croches deviennent croches + triolet de doubles. } \\
\text { - sib devient do. }\end{array}$ \\
\hline m. 36 & - pno : second groupe de doubles croches supprimé. \\
\hline m. 37 & - pno : liaison supprimée. \\
\hline m. 39 & - pno : dernière double croche supprimée. \\
\hline m. 40 & - fl : liaison du fa\# supprimée. \\
\hline m. 41 & $\begin{array}{l}\text { - pno : dernière double croche : sol\# devient sib. } \\
\text { - pno : Clé de sol barrée à la main gauche. }\end{array}$ \\
\hline m. 42 & - correction du second groupe de doubles croches [flou]. \\
\hline m. 46 & $\begin{array}{l}\text { - } 3^{e} \text { croche : fa\# devient sol. } \\
\text { - pno : } 6 \text { croches séparées } 3+3\end{array}$ \\
\hline m. 50 & - pno : 6e croche : do octavié. \\
\hline m. 51 & - pno : si et lab octaviées. \\
\hline m. 52 & - pno : appoggiature supprimée. \\
\hline m. 53 & - pno : sol supprimé. \\
\hline m. 54 & $\begin{array}{l}\text { - cl : note changée. } \\
\text { - pno : la octavié. }\end{array}$ \\
\hline m. 56 & - pno : do\# octavié. \\
\hline m. 64 & - accord changé. \\
\hline m. 68 & - pno : liaison supprimée. \\
\hline m. 74 & - liaison coupée. \\
\hline
\end{tabular}




\section{Tableau 4}

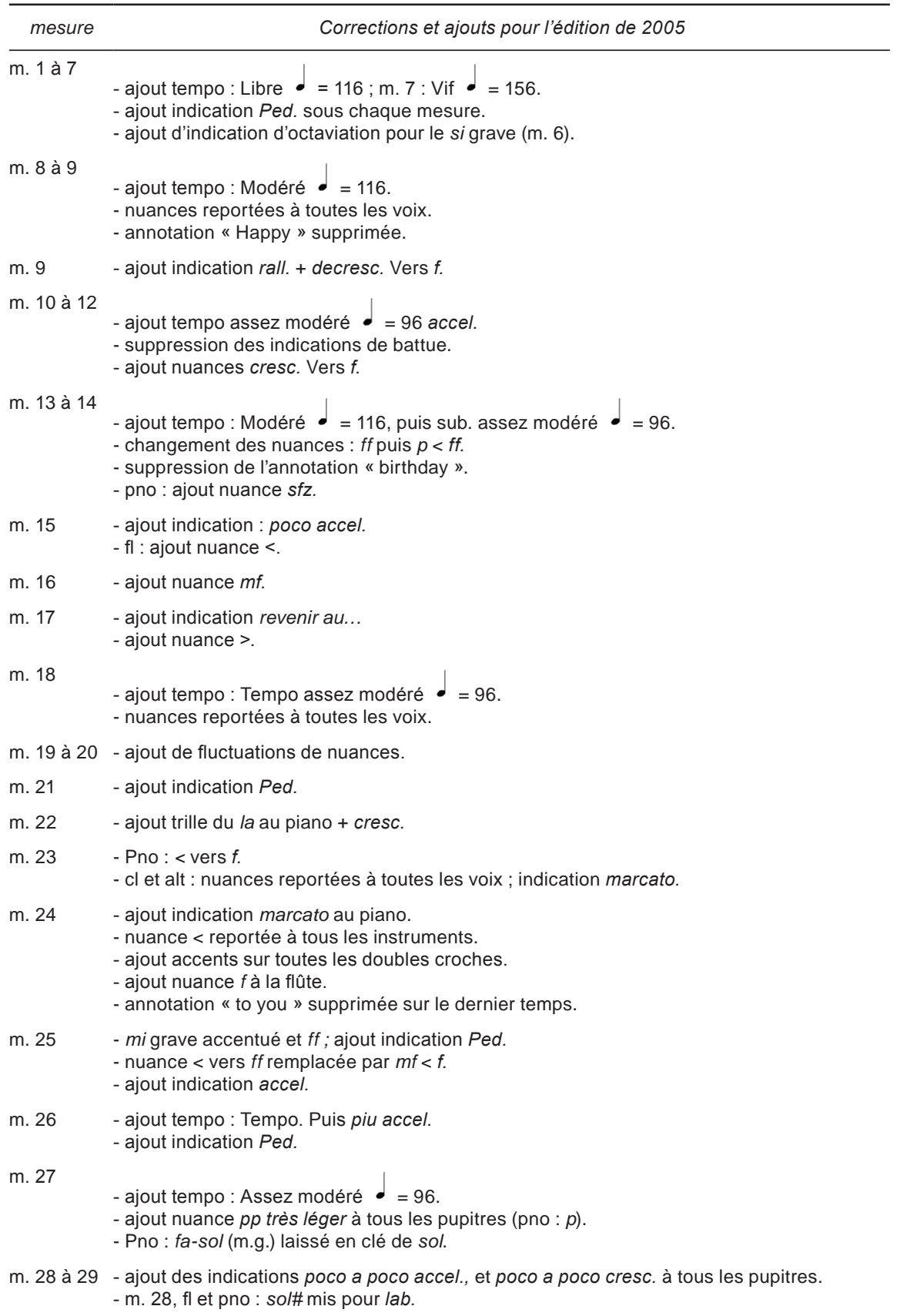




\section{Tableau 4 continué}

m. 30

- ajout tempo : Un peu plus vite $\bullet=104$.

- nuance $m p$ reportée à tous les pupitres.

m. 31 - ajout nuance < vers $m f$ à tous les pupitres.

m. 32

- ajout tempo : Revenir à ... assez modéré $\bullet=96$.

- ajout nuances $m f>$ vers $p$.

- ajout indication Ped. au piano.

- deux lignes mélodiques séparées m.d. et m.g. au piano.

m. 33 - ajout indication accel.

- croches séparées.

- ajout nuance $p<$ (fl et vc).

m. 34 - ajout nuances < vers $m f$.

m. 35

- ajout tempo : Un peu plus vite $\bullet=104$.

- ajout nuance < vers $f$.

- ajout indications Ped. au piano.

m. 36

- ajout tempo : revenir à ... Assez modéré • = 96.

- ajout nuances : > p (piano), puis $p$ à tous les pupitres.

m. 37 - pno : lab mis pour sol\# ; réb mis pour do\#.

- ajout nuances : < vers $m p ;>($ pno) ; puis $m p$ à tous les pupitres.

m. 38 - ajout tempo : poco a poco accel.

- ajout nuances : <vers $m f$; > (pno) ; puis $m f$ à tous les pupitres.

m. 39 - ajout nuances : $m f<(p n o) ;<(f l)$; puis $f$ à tous les pupitres.

- ajout indication Ped. au piano.

m. $40-\mathrm{fl}$ : altération de sécurité non reportée.

- pno : réb mis pour do\#.

- ajout nuances : $f ;>(p n o) ;<(f l)$; puis ff à tous les pupitres.

m. 41

- ajout tempo: Un peu plus vite $\bullet=104$.

- ajout nuances : $m f$, puis < (pno).

- ajout indication Ped. au piano.

m. 42 - Pno: notes redistribuées m.d. et m.g.

- ajout nuances : $f f ;>; f,<, f f($ pno) ; $f$ puis $<(f l) ; f$ à tous les autres pupitres.

m. 43 et - ajout de nuances : $f f ; m f ;<$ vers fff.

$44 \quad$ - Accent sur la dernière note à tous les pupitres.

m. 45 et

46

- ajout tempo : Un peu plus rapide $\cdot=112$.

- $\mathrm{fl}$ : ajout indication Flatt.

- inversion des nuances : $m f$ cresc. decresc. devient $m f$ decresc. $p$ cresc. (sur 2) $m f$.

m. 47 et 49

- ajout tempo: Plus rapide $\bullet=122$.

- pno : ajout indication sempre stacc.

- modification nuances : $f<>$; $><($ pno) ; $f>m f<f$ (sur 3) (autres pupitres).

m. 50 à 53

- ajout tempo : Encore plus rapide $\bullet=134$.

- modifications nuances pno : > vers $m f ; f f ;>(m .52)$; ajout nuances $f$ et $f f(m .53)$.

- ajout nuances : $f f>($ sur 3$)$ vers $m f(m .50-51)$; < vers ff (sur 3$)$ (m. 53), à tous les autres pupitres.

m. 54 - indication $p p$ reportée à tous les pupitres.

m. 55 - ajout nuance : < (sur 2). 


\section{Tableau 4 continué}

m. 56

- ajout tempo : Un peu plus modéré • = 122.

- ajout nuance < à tous les pupitres.

- pno : modification nuances : $p<m p$; mib mis pour ré\#.

m. 57

- ajout tempo : Encore plus modéré • = 112.

- ajout nuance $m p>$ à tous les pupitres.

- pno : modification nuances : $m p>$.

m. 58

- ajout tempo : Toujours plus modéré $\bullet=104$.

- nuance $p p$ à tous les pupitres.

m. 59 à 68 - suppression du texte : « Le vierge, le vivace et le bel aujourd'hui. ».

m.59

- ajout tempo : Très modéré $\bullet=104$.

- $\mathrm{fl}$ : modification nuances : $p p<$.

m. $60-\mathrm{fl}:$ modification nuances : $m p>$.

m. 61 - ajout indication Ped. (pno).

m. $62-\mathrm{cl}$ : modification nuances $p p<\left(3^{\mathrm{e}} \mathrm{tps}\right)$.

- pno : ré trille (4e tps).

- suppression du bémol de précaution ( $\mathrm{fl}$ ).

m. 63 - ajout nuances : > (cl), et $p>($ pno).

- suppression du bémol de précaution (fl).

m. 64 - ajout nuance $<(\mathrm{cl})$; changement nuances $p p$, puis $m f<f($ pno).

- ajout indication Ped.

- suppression du bémol de précaution (fl).

m. 65 - alt : app. Sol devient labb.

- nuance $m f$ reportée à tous les pupitres.

- vc : ajout nuance $m f>$.

- suppression du bémol de précaution (fl).

m. 66 - ajout nuances : $m f>(\mathrm{fl}) ;>p$ (cl et alt) $; p>(\mathrm{vc})$.

m. 67 - ajout nuances : $p p$ ( $f l$ et vc) ; > (cl et alt).

m. 68 à 73 - ajout tempo : Libre noire $=116$, puis accel. ... molto.

- cadence de piano modifiée (retranscrite ci-dessous).

- ajout indication Ped. à chaque mesure.

m. 74 à 75

- ajout tempo : vif $\bullet=156$.

- ajout nuance ff marcatissimo.

- vc : réécrit en clé d'ut.

- une coquille en partie de flûte : une noire surimprimée et de taille anormale au-dessus de la portée.

m. 76 - nuance < fff reportée à tous les pupitres.

- accents de chaque note transformés en chevrons. 


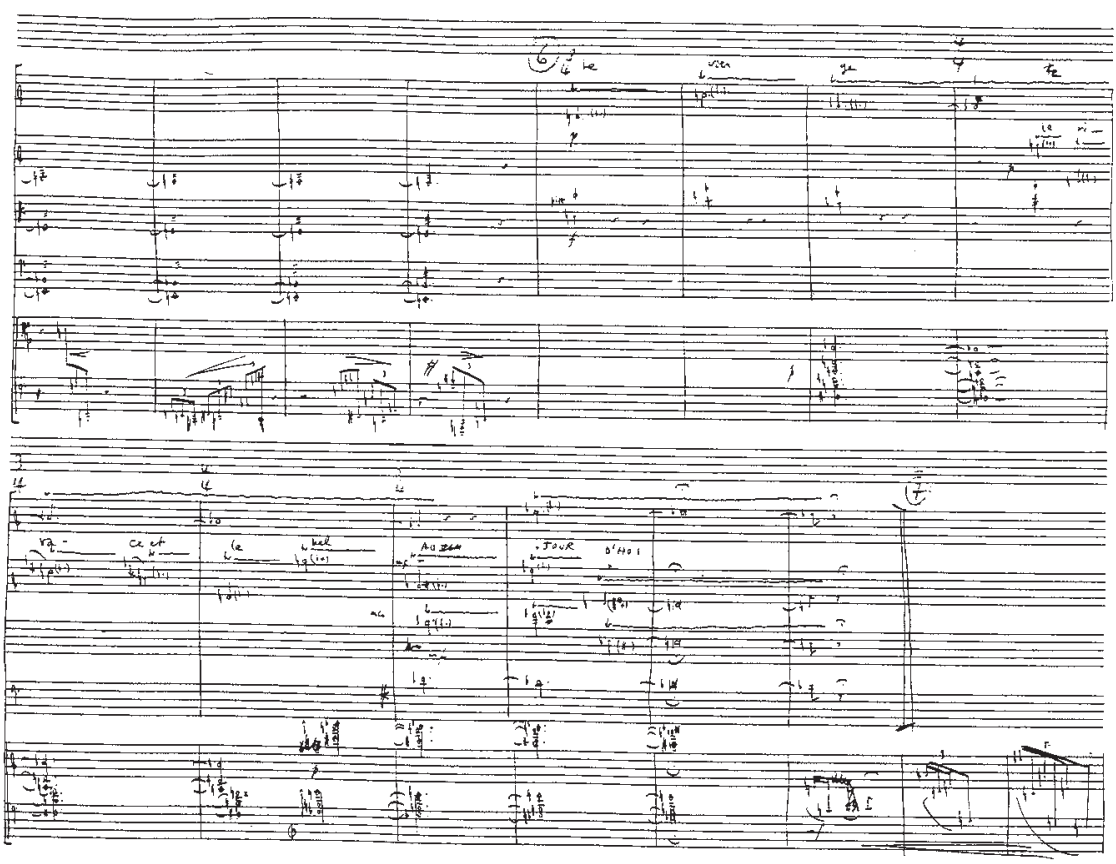

Exemple 4: 5e partie, citation de la première Improvisation sur Mallarmé. Pour le Dr. Kalmus, op. cit., p. 5 .
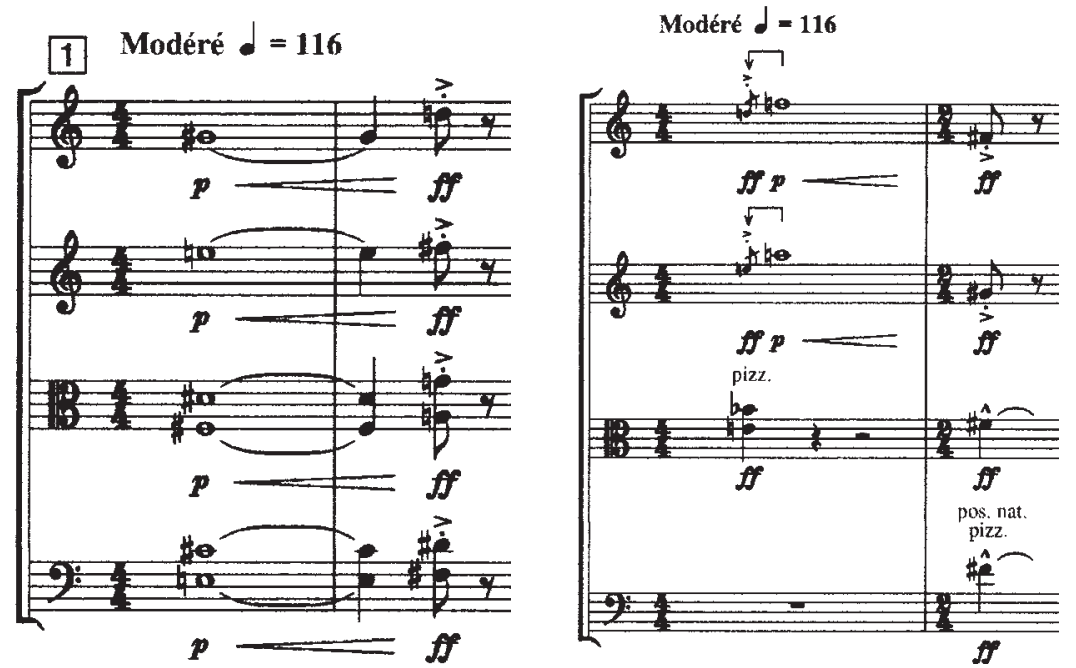

Exemples 5 a et b: interjections des vents au chiffre 1. Improvisé-pour le Dr. K., op. cit., p. 3. 


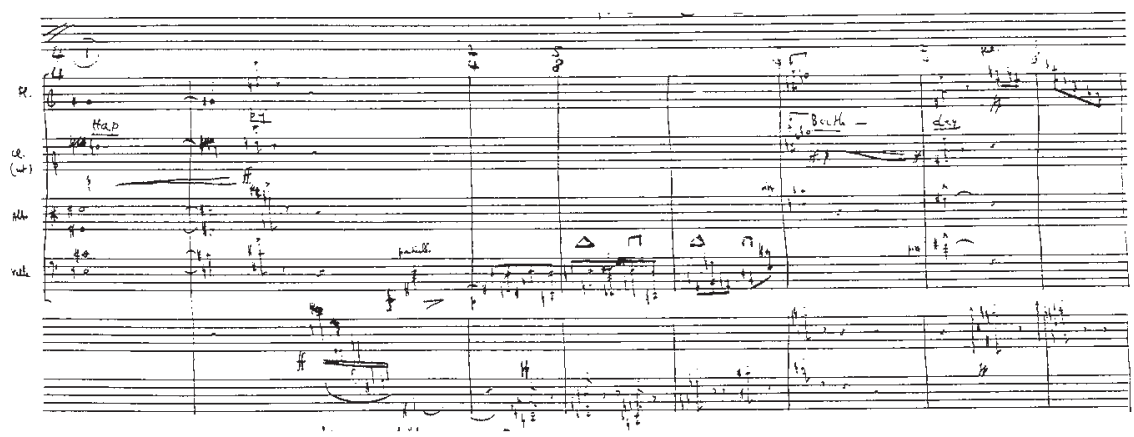

Exemple 6: citation Happy birthday to you. Pour le Dr. Kalmus, op. cit., p. 1. L'indication « to you » est rejetée à la fin de la section, m. 24 .

\begin{tabular}{|c|c|c|c|c|}
\hline & 1 & 3 & 6 & 7 \\
\hline Cadence de piano & $\begin{array}{l}\text { Happy birthday } \\
\text { to you }\end{array}$ & Partie centrale & $\begin{array}{l}\text { Improvisation I sur } \\
\text { Mallarmé }\end{array}$ & $\begin{array}{l}\text { Dév. de la cadenc } \\
\text { de piano (+ coda) }\end{array}$ \\
\hline
\end{tabular}

Exemple 7: forme générale de Pour le Dr. Kalmus

partie du matériau de la première version de Don (1960) pour voix et piano, après la tentative avortée de reprise des mêmes matériaux dans Strophes pour flûte (1957). Composée en 1965 - quatre années avant Pour le Dr. Kalmus Éclat adopte une structure simple, en cinq parties, organisées en arche autour d'une partie centrale, et avec réitération variée de la cadence initiale de piano à la fin de l'œuvre (Exemple 8).23

Toutefois, contrairement à Éclat, Tombeau, Don, Domaines et Cummings ist der Dichter ( $2^{\mathrm{e}}$ version), Pour le Dr. Kalmus est une œuvre entièrement contrôlée et préétablie. Elle ne possède aucun degré d'ouverture : ni encadrés, ni choix multiple d'intensités, ni séquences aléatoires. L’ensemble des éléments motiviques (que nous dénommons $a, b, c$, jusqu'à $g)^{24}$ de la pièce sont fixes. Par ailleurs, une projection de ces éléments dans la structure générale de l'œuvre (Exemple 9) met en évidence d'autres structures en arche, offrant ainsi plusieurs rapports entre microformes et macroforme.

\footnotetext{
23 Avec une inversion des rapports musique fixe/encadrés pour le matériau de Strophes : etc...

24 - a : trait initial « écartelé » (Exemples 3a, 3b et 10).

- b : trait(s) ascendant(s) du piano (Exemples 11a et b).

- c : quintolets (Exemples 12a et b).

- $\mathrm{d}$ : bois (Exemples 5a et b).

- e : croches appogiaturées (Exemple 16).

- f : triolets (Exemple 19).

- $\mathrm{g}$ : tenues bois (Exemple 4).
} 


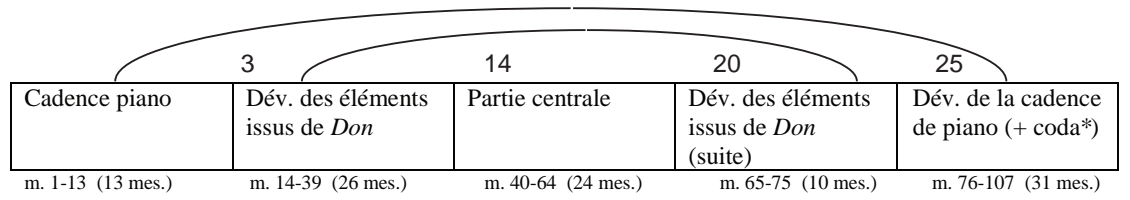

Exemple 8 : forme générale d'Éclat (1965). *Préalablement un Insert, intégré par la suite pour conclure de manière satisfaisante Eclat, puis - en cas d'enchaînement faire le lien avec Multiples. (Voir Verrier 2008, p. 38-43).

Ces structures en arche posent également la question des récurrences motiviques, alliées à l'uniformité rythmico-motivique au sein de chaque section de l'œuvre.

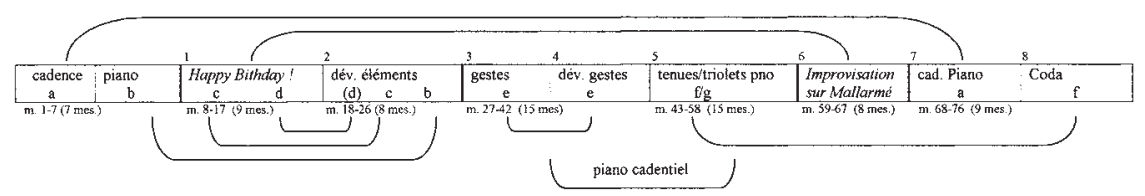

Exemple 9: structure détaillée de Pour le Dr. Kalmus

\section{RÉCURRENCES DE L'ÉCRITURE MOTIVIQUE}

On est frappé, à l'audition de Pour le Dr. Kalmus, par l'apparente clarté, au fils des structures, des récurrences stylistiques et motiviques, récurrences certes communes chez Boulez depuis les Notations et la Sonatine, mais plus évidentes ici que dans les grandes fresques que sont par exemple le Marteau sans maître et Pli selon pli25. L'un des exemples le plus frappant est le trait initial de piano, repris presque à l'identique en fin d'œuvre dans la version de 1969, et contracté en 2005 (voir Exemples 3a et 3b).

Plusieurs autres motifs sont repris, ce que l'on peut probablement justifier par la rapidité de la composition de cette pièce de circonstance. Le trait ascendant de la cadence initiale de piano (Exemple 11a) est quant à lui doublé à la fin de la première section (Exemple 11b), avec variation du nombre des hauteurs.

La récurrence est palpable également au niveau motivique. Par exemple, un court motif de quintolet, présenté isolé au piano, réapparait quelques mesures plus tard à la clarinette sib, enchaîné à l'alto ${ }^{26}$ (Exemples 12a et b).

Cette récurrence motivique était courante chez Webern - par exemple dans les cinq pièces op. 10-, il est néanmoins surprenant de constater chez Boulez une reprise à l'identique d'une formule depuis les Notations et la Sonatine.

25 Dans le Marteau, la dernière pièce («Bel édifice et des pressentiments » — le double) remémore, par fragments, des éléments des mouvements précédents; la démarche est inverse dans Pli selon pli où les trois Improvisations et Tombeau sont « annoncés » en substance dans Don (à partir de la deuxième version de 1962 pour soprano et orchestre).

26 À lire un ton en dessous (clarinette sib), et en clé d'ut 3 (alto). 


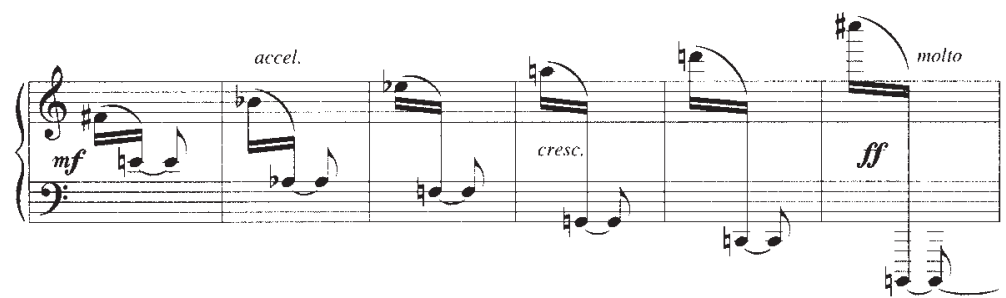

Exemple 10: trait initial. Pour le Dr. Kalmus, op. cit., p. 1.
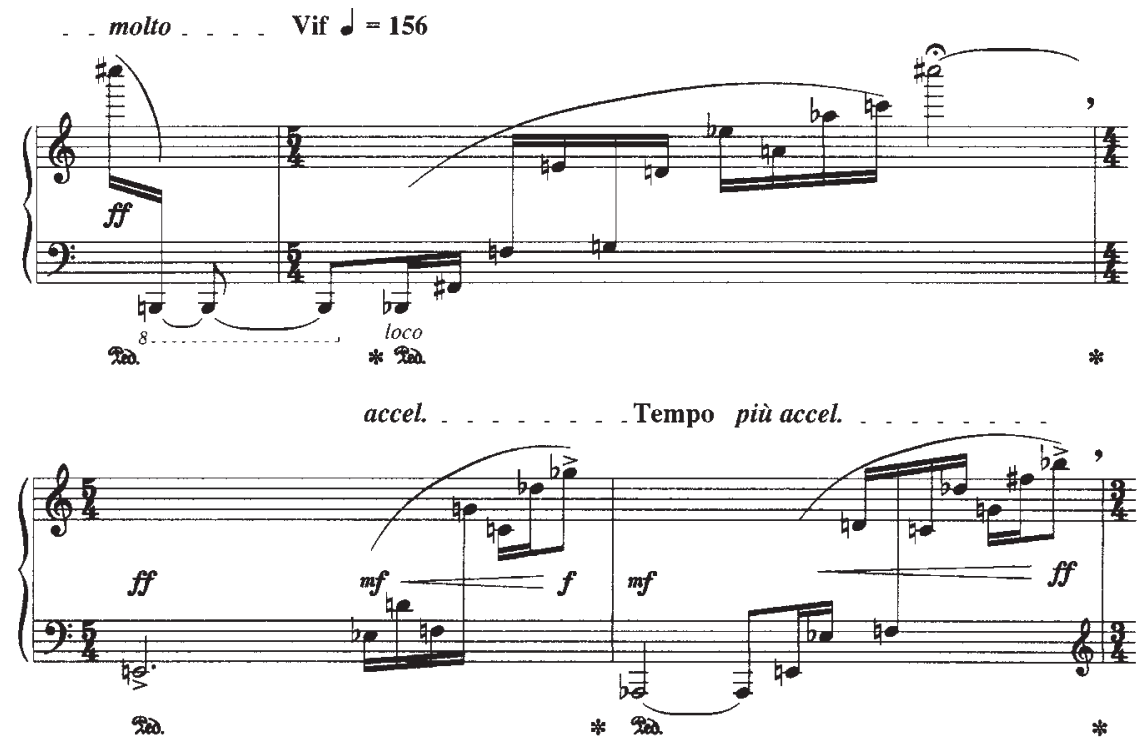

Exemples $11 \mathrm{a}$ et $\mathrm{b}$ : second trait de la cadence initiale de piano (m. 7 et 25-26). Ibid., p. 2 et 5 . 

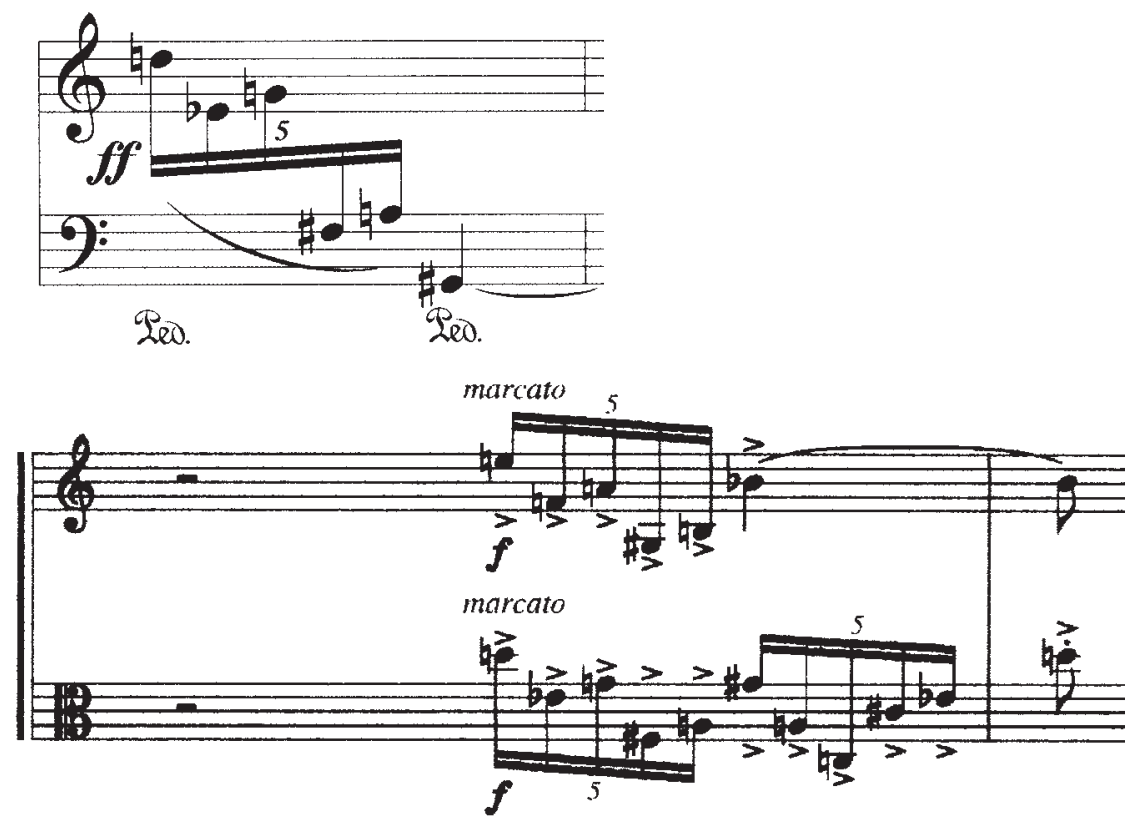

Exemples 12a et b : quintolet récurrent (m. 9 et 23-24). Ibid., p. 3 et 4.

\section{INTERVALLES ET MUTATIONS}

L'évolution des motifs au cours de l'œuvre est obtenue par un procédé que Boulez appelle «multiplication verticale» (Boulez 1963, p. 134), comparable aux jeux de mutations de l'orgue. On en trouvait déjà de nombreuses occurrences dans Le Marteau sans maître ${ }^{27}$, et notamment dans le cycle Bourreaux de solitude, où différents jeux de mutations viennent «épaissir» la polyphonie engendrée par généralisation de la série. (Koblyakov 1990, p. 35-76; et Piencikowski 1980, p. 193-266) Ce procédé est particulièrement visible dans la seconde partie de Commentaire I de Bourreaux de solitude, où sont utilisés successivement les intervalles suivants (avec leur renversement) : $3^{\mathrm{e}} \mathrm{m}$ (m. 54), $2^{\mathrm{e}} \mathrm{M}$ (m. 59), $4^{\mathrm{e} J}$ (m. 68), $9^{\mathrm{e}} \mathrm{m}(\mathrm{m} .68), 7^{\mathrm{e}} \mathrm{M}(\mathrm{m} .70)$, etc. (Exemple 13)

On retrouve ces intervalles privilégiés - plus ou moins utilisés - dans la pré-cadence d'Éclat (Exemple 7) où quatre groupes de croches reprennent en mutations successivement les quatre intervalles fondamentaux : $9^{\mathrm{e}} \mathrm{m}-7^{\mathrm{e}} \mathrm{M}-4^{\mathrm{e}} \mathrm{J}-$ $2^{\mathrm{e}} \mathrm{M}$ (Exemple 14).

Ces mêmes intervalles sont omniprésents dans Pour le Dr. Kalmus. Par exemple la neuvième majeure, mélodique, puis en mutations (Figure 15a), et la seconde majeure en mutations au piano (Exemple 15b).

Ce procédé est utilisé dès le premier trait de la cadence initiale de piano, uniquement fondé sur la seconde majeure (Exemple 10); il est également utilisé pour compléter le retour de la cadence de piano en conclusion (Exemples za et

27 Certains aspects de l'écriture harmonique des trois Psalmodies de 1945 peuvent également s'apparenter à ce procédé. 


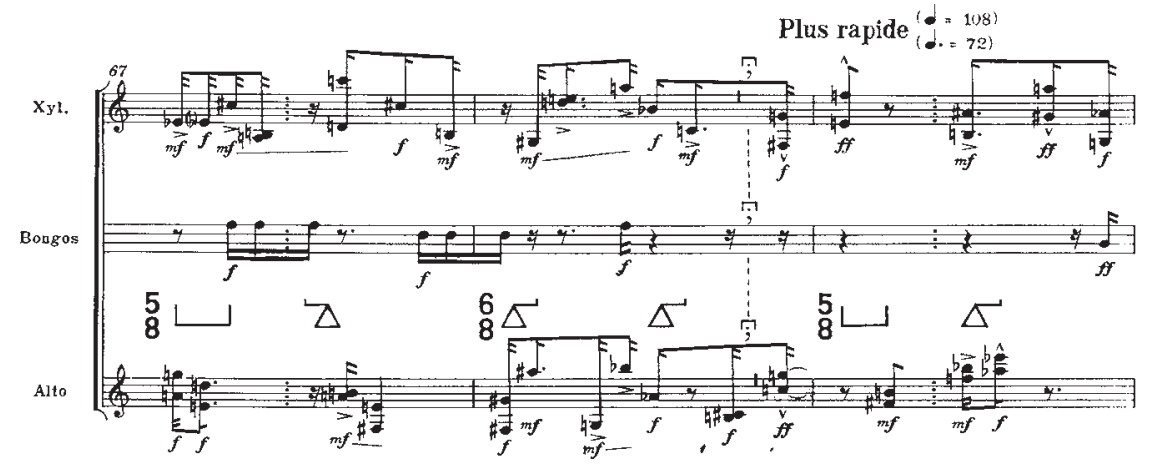

Exemple 13: extrait de Commentaire I de "Bourreaux de solitude" (m. 67 à 69). (Boulez 1957, p. 13)

2 Très rapide $d=144$

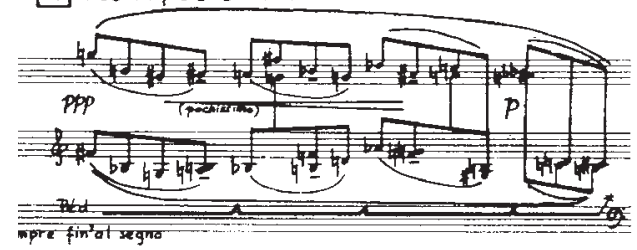

Exemple 14: extrait de la pré-cadence d'Éclat. (Boulez 1965, p. 1)

3b). Les gestes musicaux (anacrouse/croche) de la partie centrale épousent pour la plupart l'une de ces quatre structures intervalliques (Exemple 16).

Cette écriture en brefs gestes musicaux (anacrouse/croche) n'est pas propre à Kalmus. Elle se retrouve par exemple dans le «Sextuor pour cordes» de Domaines, dans la version pour clarinette et groupes instrumentaux. La partie d'alto 2 y est déduite de la partie d'alto 1, en mutation à la seconde majeure inférieure (Exemple 17).

Dans ces deux extraits, on peut constater que l'écriture se fonde sur un principe de champ harmonique, c'est-à-dire une fixité de chaque hauteur à une octaviation précise. Six sons sont utilisés pour le sextuor de Domaines (m. 1 à 4, du grave à l'aigu : sol-sib-do-fa\#-si), qui sont issus de l'un des fragments du cahier B pour clarinette seule correspondant. Dans le présent exemple issu de Kalmus, le champ harmonique est plus vaste (Exemple 18).

Pour autant, l'écriture n'est pas libre, que ce soit au niveau de la répartition de ces hauteurs ${ }^{28}$, ou des proportions des différents groupes ou différentes sections. Et ce rapprochement d'extraits de Kalmus et Domaines n'est pas illégitime - nous y reviendrons.

28 Voir par exemple, dans ma propre thèse, l'esquisse préparatoire de la strophe V de Dialogue de l'ombre double, également constituée de gestes anacrouse/croche. La rigueur est extrême dans la sérialisation des hauteurs, tant dans les notes principales que dans les anacrouses. (Tissier 2011). 


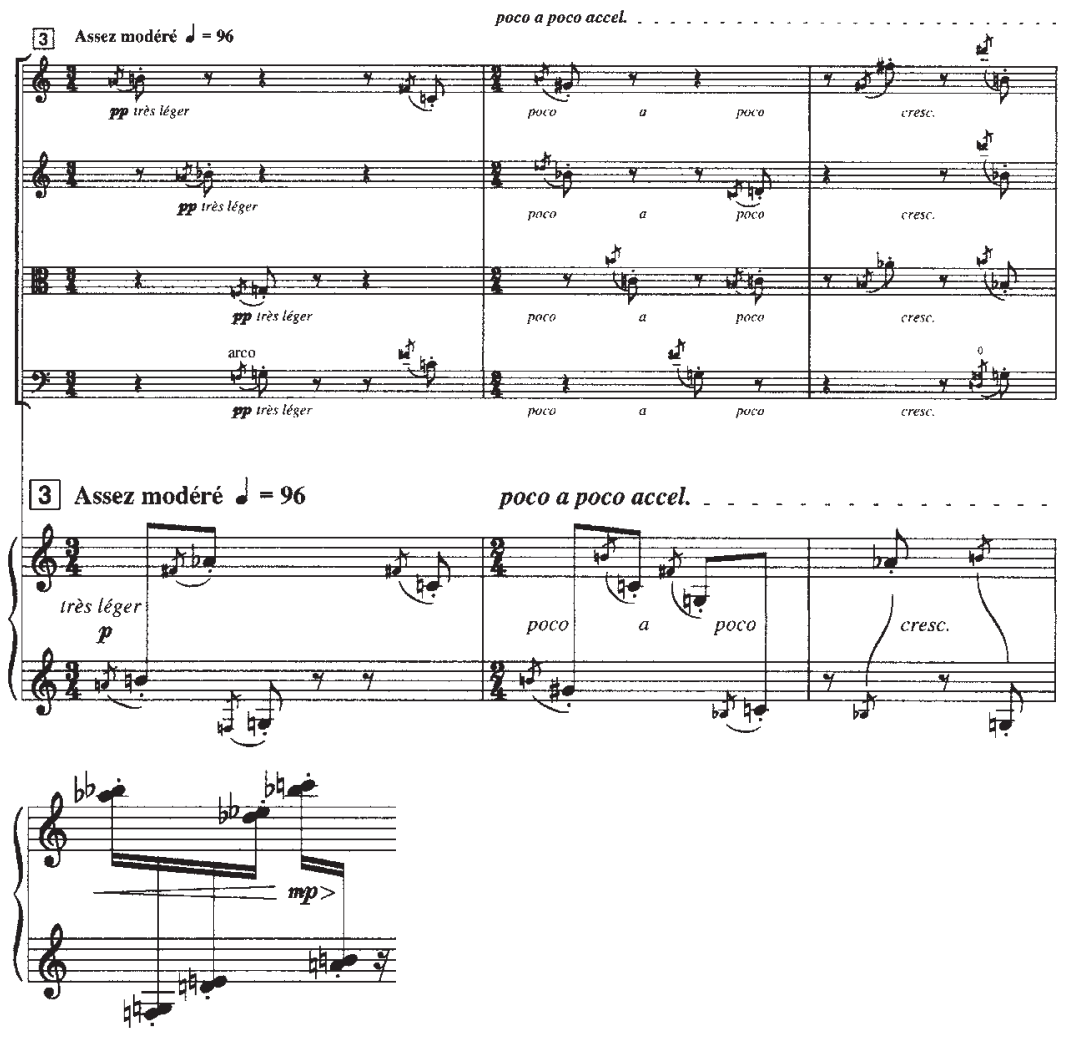

Exemples 15a et b: Improvisé-pour le Dr. K. (m.34-36, et m. 37). Op. cit., p. 6 et 7.

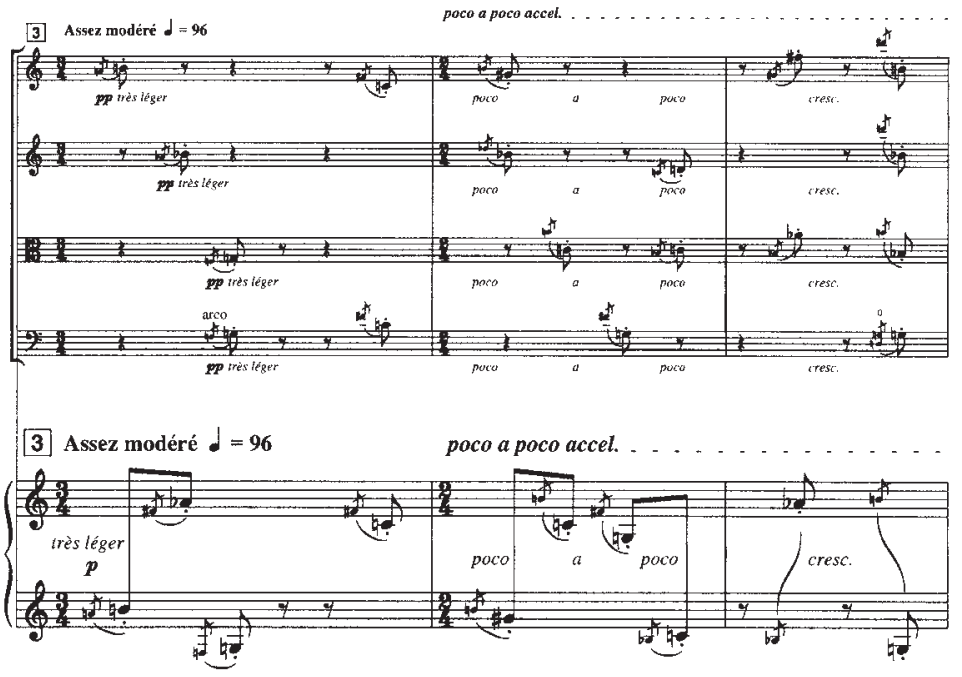

Exemple 16: Improvisé - pour le Dr. K. (m. 27-29). Ibid., p. 5. 


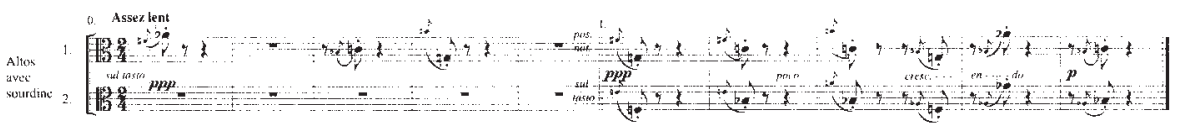

Exemple 17: «Sextuor pour cordes » (cahier B, Original) issu de Domaines

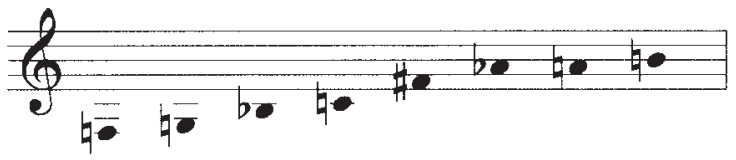

Exemple 18: champ harmonique dans la section centrale (m. 27 à 31)

\section{ORGANISATION DES PROPORTIONS}

L’analyse numérique des données de chaque section permet de démontrer que la rigueur reste omniprésente, y compris dans les proportions de chacune de ces sections et de chacun des motifs constitutifs. Par exemple, la seconde séquence de la partie centrale - succédant aux gestes anacrouse/croche précédemment évoqués - oppose deux éléments : des blocs tenus aux bois/cordes, et des arabesques en triolets au piano (Exemple 19).

Les arabesques du piano sont à densité variable, de 3 à 12 croches chacune. La succession est la suivante : 12-9-6-3-9-6-12-6-3-9-6-3, que l'on peut résumer en quatre groupes de densité décroissante, soit, par rapport à un carré structurel :

$\begin{array}{llll}12 & 9 & 6 & 3 \\ 12 & 9 & 6 & 3 \\ 12 & 9 & 6 & 3 \\ 12 & 9 & 6 & 3\end{array}$

Ce qui donne, pour les proportions $12=2 ; 9=3 ; 6=4 ; 3=3$, soit 2-3-43 (verticalement), et pour le nombre de groupes pour chaque ligne : 4-2-3-3 (horizontalement). C’est-à-dire un rapport numérique commun (2-3-3-4) mais permuté entre la dimension horizontale et la dimension verticale.

\section{Pourquoi Pour le Dr. K. - improvisé ?}

Ces considérations musicales expliquent certainement l'ajout du terme «Improvisé » au titre initial. En effet, la simplicité des procédés et de leur présentation, la clarté de la forme ainsi que des différents éléments constitutifs - justifiés par un arrangement rapide d'éléments simples - contrastent avec la richesse et la diversité de partitions plus amples alors en chantier, comme Domaines. Le constat que l'on tire de l'analyse de Pour le Dr. Kalmus renvoie ainsi à une autre œuvre de circonstance, elle aussi retirée : Symphonie mécanique pour bande, que Boulez avait alors définie à Stockhausen en ces termes :

Je vous écris après le grand mouvement de ces dernières semaines. J'avais à faire - en musique concrète, exactement déformation d'éléments naturels - la musique d'un petit film de 12' qui est un montage de mouvement 
sur trois écrans. Trop peu de temps, malheureusement, pour ne faire qu'une sorte d'improvisation préparée ${ }^{29}$.

L'autre aspect de l'improvisation concerne peut-être le rapport poḯtique existant entre matériaux bruts, préalables, et les stratégies compositionnelles choisies par Boulez pour traiter ces matériaux et les mettre en forme: plusieurs chutes réassemblées, à la manière des improvisations concertantes, mais aussi précisément des Improvisations sur Mallarmé.

Boulez réitérera cette stratégie en 2007 avec Une page d'éphéméride... pour piano, composée en rassemblant plusieurs matériaux issus de chutes d'origines diverses ${ }^{30}$. La structure par juxtaposition de séquences renforce par ailleurs cette hypothèse, à la différence d'autres œuvres pensées soit selon un processus évolutionnel (Tombeau, Rituel), soit sur une alternance régulière de séquences contrastées (Sur Incises, Répons, ...explosante-fixe...).

\section{CE QUE L'ON NE SAIT PAS ENCORE : ORIGINES ET MATÉRIAUX}

Nous avons évoqué la parenté formelle entre Pour le Dr. Kalmus et Éclat. Nous avons également évoqué la trajectoire de l'œuvre, issue vraisemblablement d'un solo de clarinette joué en avril 1969. Dans The Musical Times, William
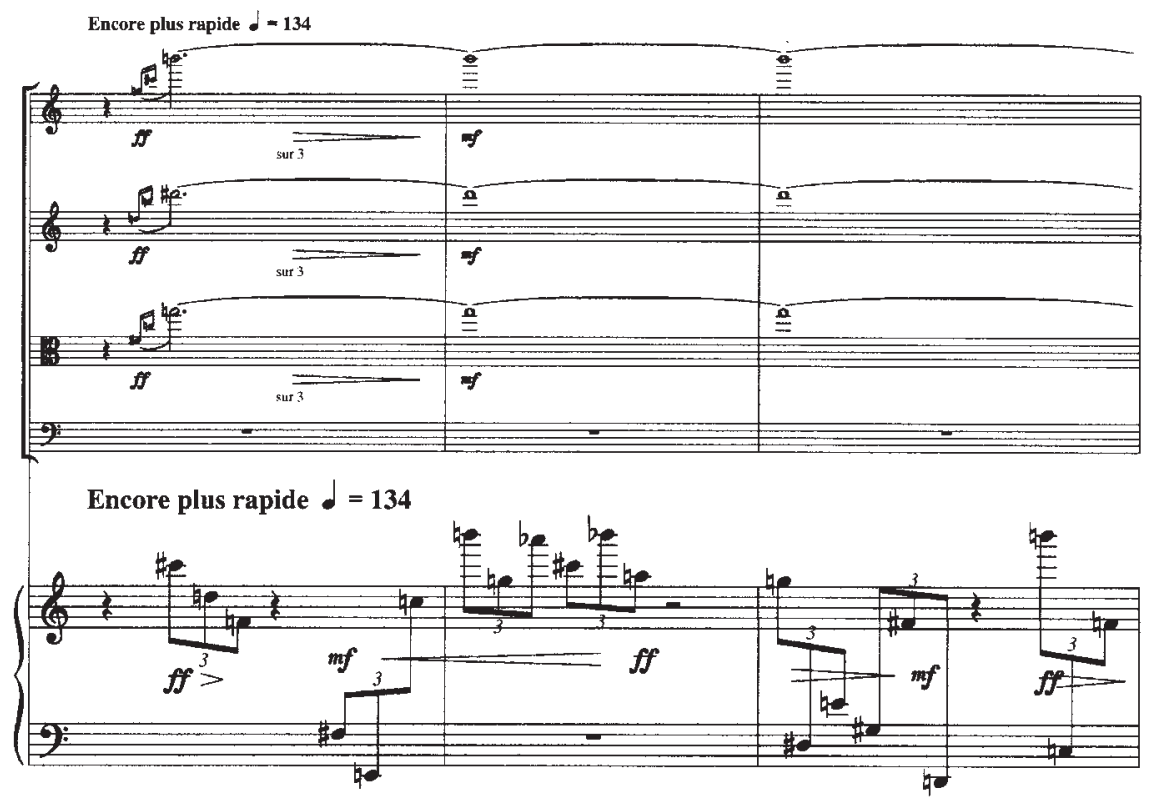

Exemple 19: extrait de la partie centrale (m. 50 à 52). Improvisé - Pour le Dr. K., op. cit., p. 9.

29 Lettre de Boulez à Stockhausen, manuscrite, non datée (cachet du 16.12.55); conservée à la Stockhausen Stiftung für Musik, Kürten. Une transcription est consultable à la Fondation Paul Sacher à Bâle.

30 Communication personnelle. 
Mann apporte une précision à son propre commentaire du Times : «Boulez wrote a clarinet solo for Alan Hacker ${ }^{31}$ that seemed to have escaped from his peripatetic Domaines. » (Mann 1969b).

Si elle ne peut à ce jour être confirmée, la proposition d'une filiation entre Domaines et Pour le Dr. Kalmus est chronologiquement plausible. Le travail sur les trois premières versions de Domaines est contemporain des deux premières versions de Pour le Dr. Kalmus : la partition pour clarinette seule (Domaines I) est créée en Allemagne par Hans Deinzer le 20 septembre 1968 à Ulm, et la première version pour clarinette et ensemble (Domaines II) deux mois plus tard à Bruxelles par Walter Boeykens, le 20 décembre 1968, avec l'Orchestre Symphonique de la Radio Belge, sous la direction du compositeur. En 1969, l'œuvre est corrigée (Domaines III), recréée par Michel Portal avec l'ensemble Musique Vivante dirigé par Diego Masson le 10 novembre 1970 au Palais de Chaillot, puis enregistrée dans la foulée ${ }^{32}$. Pour le Dr. Kalmus s'insert donc entre les versions II et III de Domaines.

Par ailleurs, il est possible de réduire toute la partition de Pour le Dr. Kalmus en une seule partie monodique, et nous avons vu que l'hommage sur «Happy birthday to you » était précisément situé à la partie de clarinette. On sait également que les matériaux de Domaines, issus de la Troisième sonate pour piano, ont donné parallèlement d'autres prolongements. Pascal Decroupet avait mis en évidence la réutilisation des tables de multiplication du «Formant $\mathrm{V}$ » (Troisième sonate), dans Domaines, pour l'élaboration de Doubles, puis de Figures, doubles, Prismes en 1957 (Decroupet 2006, p. 133-158). Ces tables seront également reprises (avec les permutations rythmiques de Constellation-miroir) pour Marges (1962-1964), tables qui, en 1955, se trouvaient déjà dans le Chæur B d'Agamemnon, extrait de l'Orestie et repris en 1957 comme prélude à la Deuxième improvisation sur Mallarmé.

Les origines de l'hommage à Kalmus demeurent quoi qu'il en soit incertaines, et il paraît illusoire d'espérer, par l'analyse des données intrinsèques des partitions publiées en 1969 et 2005, une vérité sur les contenus matériels. Il faudra probablement attendre le dépôt des éventuels documents - esquisses et manuscrits - à la Fondation Paul Sacher pour démêler les liens matériels unissant hypothétiquement Pour le Dr. Kalmus aux différentes œuvres évoquées : Éclat, Domaines et les Improvisations sur Mallarmé.

\section{Conclusion}

Puisque de nombreux points obscurs subsistent concernant cette œuvre, les présentes considérations sur Pour le Dr. Kalmus appellent désormais un travail sur les arcanes internes de l'œuvre, afin d'en décrypter le contenu, ainsi que les matériaux d'origine.

Si l'on ignore encore les raisons qui ont convaincu Boulez de reconnaître son bref hommage, il est important de replacer cet évènement dans le contexte

31 Clarinettiste, cofondateur des Pierrot players, Alan Hacker est décédé le 16 avril 2012, avant d'avoir pu répondre à notre demande de renseignements sur la création du 22 avril 1969.

32 Domaines, Harmonia mundi 55930, 1971. 
de ces vingt dernières années, car la renaissance de Pour le Docteur Kalmus en 2005 n'est pas un cas isolé. Au crépuscule de son répertoire, Boulez semble souhaiter revenir sur certains inachevés. Le compositeur, emblématique du Work in progress, dont on connaît aujourd'hui l'ampleur des ramifications labyrinthiques des matériaux, poursuit une politique d'achèvement de ses œuvres, mais aussi d'acceptation progressive d'une certaine forme de transgression, de propagation de son œuvre au-delà des gels idéologiques et morphologiques.

Dans les années 1980, il s'agissait de donner une forme et une reconnaissance aux œuvres de jeunesse maintes fois triturées et révisées au fil des évolutions esthétiques et des révolutions techniques; en 2010, par exemple, Le Visage nuptial - pourtant une œuvre secondaire de la production boulézienne - accédait, par un regard rétrospectif de l'auteur, à un statut d'œuvre phare : «Je m'y suis étendu longuement parce que c'est une œuvre pour moi qui a marqué des jalons dans mon expérience musicale, et qui marque tout de même une permanence dans ma relation au poétique ${ }^{33}$."

De manière plus surprenante, en 2006, Boulez autorise l'extraction et l'interprétation indépendante de deux solos de flûte en sol issus du Marteau sans maître, à la demande d'Aurèle Nicolet pour l'Académie du Festival de Lucerne 2007. La partition sera publiée sous le titre Deux extraits du Marteau sans maître. Études pour flûte alto (1953). Cette pratique était plutôt l'apanage de Stockhausen, avec l'adaptation constante des pièces aux différents pupitres instrumentaux.

D'autres œuvres connaitront une translation vers d'autres instruments : Messagesquisse pour sept violoncelles existe depuis 2000 en une version pour sept altos ${ }^{34}$. Le Dialogue de l'ombre double aura connu quant à lui quatre versions à ce jour. À la version première pour clarinette s'ajoutent désormais les versions pour basson ${ }^{35}$, saxophones ${ }^{36}$ et flûte ${ }^{37}$. Dernièrement, c'est Anthèmes (1992) et Anthèmes 2 (1997) qui furent transposés afin d'être adaptés pour l'alto (2008).

Citons encore la seule orchestration de Boulez réalisée en 1987 de Frontispice de Maurice Ravel, et dont on a appris récemment l'adaptation pour grand orchestre en 2007. Les deux partitions sont dorénavant disponibles chez Universal Edition.

En novembre et décembre 2012, c'est le Livre pour quatuor qui devait connaître un achèvement. Il y a douze ans, le Quatuor Parisii avait déjà réalisé une révision, ainsi qu'un enregistrement du Livre, revu et corrigé par Boulez; mais demeurait encore inédit le fameux mouvement IV, absent de l'édition de 1960. Prévue le 10 décembre 2012, sa création fut hélas une fois encore repoussée.

\footnotetext{
33 P. Boulez, entretien avec Claude Lefebvre, Université Paul Verlaine - Metz, 2010.

34 Messagesquisse, version pour 7 altos (1976/2000), Londres, Universal Edition UE 31700, 2000, $28 \mathrm{p}$.

35 Suggérée par Pascal Gallois, bassoniste de l'Ensemble InterContemporain. Identique à l'originelle, la partition n'est que transposée. Elle est créée par Pascal Gallois le 3 novembre 1995.

36 Créée le 23 juin 2001 au Théâtre des Bouffes du Nord par Vincent David. Pour des raisons d'ambitus, le jeu du Dialogue au saxophone nécessite plusieurs instruments.

37 Créé par Cécile Daroux en mai 2002 à San Francisco.
} 
Comme Pour le Dr. Kalmus, certaines pages de Boulez conservent donc encore, pour quelques temps, leur part de mystère.

\section{RÉFÉRENCES}

Baltensperger, André. 1990. "Marginalien zu einem Manuskript von Pierre Boulez», Mitteilungen der Paul Sacher Stiftung n³, 15-22. Bâle: Fondation Paul Sacher.

Boulez, Pierre. 1995. "Construire une improvisation». Points de repère I/ Imaginer, sous la dir. de Jean-Jacques Nattiez, 445-466. Paris : Christian Bourgois.

—.1987. Penser la musique aujourd'hui. Paris : Gallimard. (Publ. orig. Paris : Denoël/Gonthier, 1963.)

-1965. Éclat/Multiples pour orchestre @ Copyright 1965 by Universal Edition (London) Ltd., London/UE 18296.

_. 1957. Le Marteau sans maître, (c) Copyright 1957 by Universal Edition (London) Ltd., London/UE12450.

Dal Molin, Paolo. 2007. "Introduction à la famille d'œuvres ...explosantefixe... de Pierre Boulez. Etude philologique.» Thèse de doctorat, Université de Nice - Sophia Antipolis.

Decroupet, Pascal. 2006. "Moments doubles, figurés en prismes». Pierre Boulez. Techniques d'écriture et enjeux compositionnels, sous la dir. de Pascal Decroupet et Jean-Louis Leleu, 133-158. Genève : Contrechamps Éditions.

Hopkins, G. W. et Paul Griffiths. 2001. «Boulez, Pierre». The New Grove Dictionary of Music and Musicians, seconde édition. Londres : Macmillan Publishers.

Jameux, Dominique. 1984. Pierre Boulez. Paris : Fayard.

Koblyakov, Lev. 1990. Pierre Boulez. a world of harmony, Vol. 2. Londres: Osborne.

Mann, William. 1969a. "A Garland for Dr. Kalmus». The Times, vendredi 23 avril : 16 .

—1969b. «Music in London: Birthday Concerts». The Musical Times 110, $n^{\circ} 1516: 645$.

Piencikowski, Robert. 1980. "René Char et Pierre Boulez. Esquisse analytique sur le Marteau sans Maître». Schweizer Beiträge zur Musikwissenschaft, vol. IV, 193-266. Bern \& Stuttgart : Verlag Paul Paupt.

- 1985. «Nature morte avec guitare». Pierre Boulez. Ein Festschrift für 60. Geburtstag am 26. März 1985, sous la dir. de Joseph Häusler, 66-81.Vienne : Universal Edition.

—.1993. "'assez lent, suspendu, comme imprévisible' : quelques aperçus sur les travaux d'Éclat». Écritures musicales aujourd'hui, Genesis, no 4 : 51-68. Paris : Jean-Michel Place.

Tissier, Brice. 2011. «Mutations esthétiques, mais continuité technique». Thèse de doctorat, Université de Montréal/Université de Paris Sorbonne.

Verrier, Mickael. 2008. "Un fragment chez Boulez». Mitteilungen der Paul Sacher Stiftung n²1 : 38-4. Bâle : Paul Sacher Stiftung. 


\title{
DiscographiE
}

Pour le Dr. Kalmus. 1970. Dans A Garland for Dr. K. Compositions for the 8oth birthday of Dr. Alfred A. Kalmus (hors commerce), Vicente Sempere Gomis (fl), José Vadillo (cl), Pablo Ceballos Gomez (vla), Angel Gonzalez Quinones (vc), Maria Manuela Caro De Halffter (pno). Londres : Universal Edition UE 15043-104.

Eclat. 1990. Dans Rituel/Eclat/Multiples. BBC Symphony Orchestra, Ensemble InterContemporain, dir. Pierre Boulez. Sony SMK 45839.

\section{RÉSUMÉ}

Retirée après sa création en 1969, la pièce Pour le Dr Kalmus de Pierre Boulez a fait en 2005 une surprenante réapparition. On ignore encore les raisons qui ont décidé Boulez à réviser cette partition. On ignore d'ailleurs presque tout la concernant. Il est toutefois possible aujourd'hui, à la lumière des quelques sources manuscrites conservées et de la nouvelle partition éditée, de faire plusieurs constatations historiques, philologiques et analytiques sur cette œuvre certes secondaire dans le corpus boulézien, mais non dénuée d'intérêt, et composée à un moment charnière de sa production musicale et de son évolution esthétique.

\begin{abstract}
Removed after its creation in 1969, Pierre Boulez's work Pour le Dr. Kalmus, reappeared surprisingly in 2005. The reasons why Pierre Boulez revised this work are still ignored, as is also almost everything about it. However, it is possible today to learn more from the few remaining manuscript sources and the revised score. Their study allows some historical, philological and analytical observations on this work, secondary in Boulez's work, but not uninteresting, since it was composed at a pivotal moment in Boulez's musical work and aesthetic evolution.
\end{abstract}

